\title{
A SPECIAL CASE OF POSITIVITY
}

\author{
S. P. DUTTA
}

(Communicated by William C. Waterhouse)

\begin{abstract}
In this note we prove a special case of positivity of Serre's Conjecture on intersection multiplicity of modules $[\mathbf{S}]$. The conjecture can be stated as follows.
\end{abstract}

Let $R$ be a regular local ring and let $M$ and $N$ be two finitely generated modules over $R$ such that $l(M \otimes N)<\infty$. Then $\chi(M, N)=\sum_{i=0}^{\operatorname{dim} R}(-1)^{i} l\left(\operatorname{Tor}_{i}^{R}(M, N)\right) \geq 0$, the sign of inequality holds if and only if $\operatorname{dim} M+\operatorname{dim} N=\operatorname{dim} R$.

Serre proved the conjecture in the equicharacteristic and in the unramified case. Recently P. Roberts $[\mathbf{R}]$ and H. Gillet and C. Soule $[\mathbf{H}-\mathbf{G}]$ proved independently the vanishing part, i.e. $\chi(M, N)=0$ when $\operatorname{dim} M+\operatorname{dim} N<\operatorname{dim} R$ in more generality. The positivity part, i.e. $\chi(M, N)>0$ when $\operatorname{dim} M+\operatorname{dim} N=\operatorname{dim} R$ is still very much an open question.

We write $R=V\left[\left[x_{1}, \ldots, x_{n}\right]\right] /(f), V$ a complete discrete valuation ring, $p$ a generator of the maximal ideal of $V, p \in m^{2}$ where $m$ is the maximal ideal of $R$ and $f \in m-m^{2}$. We divide the whole problem into three parts:

1. $p M=0, p N=0$. This case was proved by Malliavin-Brameret $[\mathbf{M}]$.

2. $p$ is a non-zero-divisor on $M$ and $p$ is nilpotent on $N$.

3. $p$ is a non-zero-divisor on both $M$ and $N$.

The theorem which we are going to prove is the following

THEOREM. Let $R$ be a regular local ring. Let $M$ and $N$ be two finitely generated modules over $R$ such that

(i) $M$ is Cohen-Macaulay.

(ii) $l(M \otimes N)<\infty$ and $\operatorname{dim} M+\operatorname{dim} N=\operatorname{dim} R$.

(iii) $p^{t} N=0$ for some integer $t$ and $p$ is a non-zero-divisor on $M$.

Then $\chi(M, N)>0$.

The above theorem was already proved by the author in the case when $\operatorname{dim} R \leq 5$ in [D2]. The vanishing theorem of Roberts (Gillet and Soulé) and the techniques developed by the author in [D1] now make it possible to prove the above version.

PROOF OF THE THEOREM. We divide the proof into two steps.

Step 1. Let $R$ be a Gorenstein local ring of characteristic $p>0$. Let $M$ be a module of finite projective dimension and let $N$ be any other module over $R$ such that $l(M \otimes N)<\infty$ and $\operatorname{dim} M+\operatorname{dim} N \leq \operatorname{dim} R$.

Let $f: R \rightarrow R$ be the Frobenius map, i.e. $f(x)=x^{p}$. We denote by $f_{R}^{n}$ the bialgebra $R$, having the structure of an $R$-algebra from the left by $f^{n}$ and from

Received by the editors March 7, 1986 and, in revised form, February 13, 1987.

1980 Mathematics Subject Classification (1985 Revision). Primary 13H15, 13D99.

Partially supported by a NSF grant. 
the right by the identity map, i.e. if $\alpha \in R, x \in f_{R}^{n}, \alpha x=\alpha^{p^{n}} x$, and $x \alpha=x \alpha$. We assume $K=R / m$, where $m$ is the maximal ideal of $R$, is perfect. (This assumption is not at all restrictive with respect to generalized type of intersection multiplicity conjectures.) We denote by $F^{n}(M)$ the object $M \otimes f_{R}^{n}$. We define $\chi_{\infty}(M, N)=\lim _{n \rightarrow \infty} \chi\left(F^{n}(M), N\right) / p^{n \cdot \operatorname{codim} M}$. The following properties of $\chi_{\infty}$ were proved in [D1].

1. If $\operatorname{dim} M+\operatorname{dim} N<\operatorname{dim} R$, then $\chi_{\infty}(M, N)=0$ (Corollary 1, p. 437).

2. If $M$ is Cohen-Macaulay, then

$$
\chi_{\infty}(M, N)=\lim _{n \rightarrow \infty} l\left(F^{n}(M) \otimes N\right) / p^{n \cdot \operatorname{codim} M}
$$

and this is a positive integer if $R$ is a complete intersection.

3. $\chi_{\infty}(M, N)=\chi(M, N)$ if the vanishing conjecture holds for every pair $(M, T)$ with $l(M \otimes T)<\infty$ and $\operatorname{dim} M+\operatorname{dim} T<\operatorname{dim} R$ (this assertion follows easily from Proposition 1.2 of [D1]).

Step 2 . Under the hypothesis in our theorem, since $\chi$ is additive, we can assume $p N=0$. Since $p$ is a non-zero-divisor on both $R$ and $M$ and $p N=0$, we have

(i) $\chi^{R}(M, N)=\chi^{R / p R}(M / p M, N)$.

(ii) P.d. ${ }_{R / p R} M / p M<\infty$ and $\chi^{R / p R}(M / p M, T)=0$, where

$$
\operatorname{dim} M / p M+\operatorname{dim} T<\operatorname{dim} R / p R
$$

(since this implies $\operatorname{dim} M+\operatorname{dim} T<\operatorname{dim} R$, and $\chi(M, T)=0$ [G-S, R]).

(iii) $M / p M$ is Cohen-Macaulay over $R / p R$ with Ch. $R / p R=p>0$.

We denote $R / p R$ by $\bar{R}$. We have by (ii) and (3) of Step 1

$$
\chi_{\infty}^{\bar{R}}(\bar{M}, N)=\chi^{\bar{R}}(\bar{M}, N)=\chi^{R}(M, N) .
$$

Moreover by (iii) and (2) of Step 1

$$
\chi_{\infty}^{\bar{R}}(\bar{M}, N)=\lim _{n \rightarrow \infty} l\left(F^{n}(\bar{M}) \otimes N\right) / p^{n \cdot \operatorname{codim} \bar{M}} .
$$

Hence $\chi^{R}(M, N)>0$.

REMARK. Unfortunately, $\chi_{\infty}$ fails to behave like a "multiplicity function" over $R$ for pairs $(M, N)$ with P.d. $M<\infty, l(M \otimes N)<\infty, \operatorname{dim} M+\operatorname{dim} N=\operatorname{dim} R$ when $M$ is not Cohen-Macaulay. This was pointed out in [D-H-M]. The counterexample discussed there gives rise to a module $M$ with P.d. $M<\infty, \operatorname{dim} M=1$, depth $M=0$ and another module $N$ such that $\chi_{\infty}(M, N)$ is negative.

\section{REFERENCES}

[D1] S. P. Dutta, Frobenius and multiplicity, J. Algebra 85 (1983).

[D2] _ Generalized intersection multiplicities of modules, Trans. Amer. Math. Soc. 276 (1983).

[D-H-M] S. P. Dutta, M. Hochster, and J. E. McLaughlin, Modules of finite projective dimension with negative intersection multiplicities, Invent. Math. 79 (1985), 253-291.

[G-S] H. Gillet and C. Soulé, $K$ theorie et nullite des multiplicites d'intersection, C. R. Acad. Sci. Paris Ser. I 300 (1985), 71-74.

[H] M. Hochster, Conference on commutative algebra, Lecture Notes in Math., Vol. 311, SpringerVerlag, Berlin and New York, 1973, pp. 120-152. 
[M] M. P. Malliavin-Brameret, Une remarque sur les anneaux locaux reguliers, Sém. Dubreil-Pisot Algèbre et Théorie des Nombres, 24 année, 1970/71, No. 13.

[R] P. Roberts, The vanishing of intersection multiplicities of perfect complexes, Bull. Amer. Math. Soc. 13 (1985).

[S] J.-P. Serre, Algèbre locale multiplicités, Lecture Notes in Math., vol. 11, Springer-Verlag, Berlin, Heidelberg, and New York, 1957/58.

Department of Mathematics, University of Pennsylvania, Philadelphia, PENNSYLVANIA 19104-6395

Current address: Department of Mathematics, University of Illinois, Urbana, Illinois 61801 